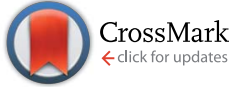

Cite this: RSC Adv., 2017, 7, 14511

11th January 2017

Accepted 21st February 2017

DOI: $10.1039 / \mathrm{c} 7 \mathrm{ra00426e}$

rsc.li/rsc-advances

\section{Endowing a triarylboron compound showing ACQ with AIE characteristics by transforming its emissive TICT state to be dark $\dagger$}

\author{
Jun Liu, ${ }^{a}$ Chenghua Zhang, ${ }^{a}$ Jun Dong, ${ }^{a}$ Jiang Zhu, ${ }^{\text {a }}$ Chengyi Shen, ${ }^{\text {*a }}$ \\ Guogiang Yang ${ }^{\star b}$ and Xiaoming Zhang ${ }^{c}$
}

A triarylboron-based AIE fluorophore, TAB-AIE-FL, was designed and synthesized by introducing an electron acceptor to boost charge separation, transforming its TICT state from bright emission to dark. We further introduced it into NIH/3T3 cells using nanogel as a carrier for imaging the intracellular environments of cytoplasm and nucleus.
$\pi$-Conjugated triarylboron compounds have aroused much interest for various optical and electronic applications. ${ }^{1,2}$ Due to its vacant $\mathrm{p}_{z}$ orbital, boron can be used as a strong p-electron acceptor when connected to electron donor groups and an intramolecular charge transfer (ICT) can result, which usually demonstrates strong fluorescence. Based on this principle, some triarylboron-based fluorophores have been developed both by others and our group..$^{3-7}$ Considering their molecular structure can be modified to achieve a diversity of functions, recently they have also been successfully applied to the area of bio- and chemosensing by others and by us. ${ }^{8-12}$ Some triarylboron-based biological fluorescence probes have been developed, including imaging for intracellular temperature, ATP, $\mathrm{H}_{2} \mathrm{~S}$, biothiol and mitochondria etc. Although they possess some excellent photophysical properties, including a large two-photon crosssection $^{8,12}$ and high quantum yields, and are extremely sensitive to changes in their surroundings, ${ }^{6,7,9,10}$ we realized that in the experimental process, they usually demonstrate stronger fluorescence in solution than in the solid state; they suffer from the same aggregation-caused quenching (ACQ) problem with some traditional fluorophores..$^{13}$ Therefore, to overcome this drawback and further expand their applications, it is urgent for us to develop some triarylboron fluorophores showing stronger fluorescence in the solid state than in solution.

The concept of aggregation-induced emission (AIE), where non-emissive luminogens are induced to emit by aggregate

${ }^{a}$ Sichuan Key Laboratory of Medical Imaging, Department of Chemistry, School of Preclinical Medicine, North Sichuan Medical College, Nanchong, 637000, China. E-mail: jzhunsmc@hotmail.com; shchengy@126.com

${ }^{b}$ Beijing National Laboratory for Molecular Sciences, Key Laboratory of Photochemistry, Institute of Chemistry, Chinese Academy of Sciences, Beijing 100190, China.E-mail: gqyang@iccas.ac.cn

${ }^{c}$ Sichuan Key Laboratory of Medical Imaging, Department of Radiology, Affiliated Hospital of North Sichuan Medical College, Nanchong, 637000, China

$\dagger$ Electronic supplementary information (ESI) available. See DOI: $10.1039 / \mathrm{c} 7 \mathrm{ra00426e}$ formation, was first proposed by the group of Tang in $2001 .^{14}$ Compared to the traditional aggregation-caused quenching (ACQ) of fluorophores, the AIE fluorophores experience a totally opposite luminescence process, that is demonstrating strong fluorescence in the solid state but almost no fluorescence in solution. ${ }^{15}$ Since the first report on AIE phenomena was published, AIE chromophores have received considerable attention, and numerous AIE luminogens have been developed in the past decade. ${ }^{16}$ The AIE processes have also been applied in a number of fields and possess some advantages, for example AIE-based fluorophore probes can improve photostability and signal intensity. ${ }^{17-19}$ Therefore, endowing triarylboron compounds with AIE properties is a possible approach for us to promote their performance in various applications.

In order to understand the AIE phenomena, a number of mechanistic pathways have been hypothesized. ${ }^{20}$ Among these explanations, there is much solid proof to show that a non fluorescent twisted intramolecular charge transfer (TICT) process can contribute to fluorescence enhancement of the AIE process. ${ }^{21,22}$ As our previous studies have demonstrated, the strong fluorescence emission of triarylboron compounds is usually attributed to two distinct excited states, the local excited state (LE) and the TICT excited state. ${ }^{6,7}$ Therefore, if we can transform the TICT state of triarylboron compounds from strong emission to a dark state, the goal of developing a triarylboron-based AIE fluorophore may be achieved. This achievement can also expand the application of triarylboron compounds in some fields.

In order to boost the faint TICT process of triarylboron compounds, an electron acceptor should be introduced to further increase their ICT and even boost their total charge separation. ${ }^{23}$ The introduced acceptor should also be rotatable so that it can further enhance the degree of molecular twisting during the charge separation and lead to a weak emissive TICT state. ${ }^{24}$ Moreover, the rotatable acceptor, whose number and 
rotor amplitudes do not need to be very large, according some previous reports, can dissipate the excited-state energy by bringing about intramolecular rotation. ${ }^{25}$

With these ideas in mind, a triarylboron-based AIE fluorophore, TAB-AIE-FL, was designed and synthesized by introducing an electron withdrawing group, 2,4-di-tert-butyl-striazine, to the structure of a triarylboron compound (Scheme 1). The two tert-butyls with a large volume are helpful for blocking $\pi-\pi$ stacking interaction in the aggregates. As a contrast, a traditional triarylboron compound, TAB-2-PR, was also synthesized. The AIE phenomena of TAB-AIE-FL was confirmed by measurement of the spectrum. Theoretical investigations also indicate that there exist two excited states for TAB-AIE-FL, corresponding to its LE excited state and its nonemissive TICT excited state. We also studied the comprehensive effects of viscosity and polarity on the luminescence of monodispersed TAB-AIE-FL, which further confirm that the AIE mechanism of TAB-AIE-FL involves restriction of intramolecular rotation (RIR) and also shows that TAB-AIE-FL can be used as a "turn on" fluorescence probe for viscosity. On incorporating TAB-AIE-FL into the polyurethane nanogel (NG-TAB-AIE-FL), strong fluorescence was observed, which can be attributed to the high viscosity and low polarity in the hydrophobic domains or the formation of aggregates in the hydrophilic domains. TABAIE-FL was also introduced into NIH/3T3 cells using polyurethane nanogel as a carrier. The bright fluorescence in cells also resulted from the low polarity and high viscosity environment of the nucleus and the formation of aggregates in the hydrophilic environment of the cytoplasm, which can be clearly seen by confocal imaging.

The synthesis and characterization of TAB-2-PR and TABAIE-FL are recorded in the ESI. $\dagger$ TAB-2-PR and TAB-AIE-FL are highly soluble in common organic solvents, including $n$-hexane, ethyl acetate, THF and ethanol, but they exhibit poor solubility in water. The absorption and emission spectra of TAB-2-PR and TAB-AIE-FL were measured in solvents of different polarity

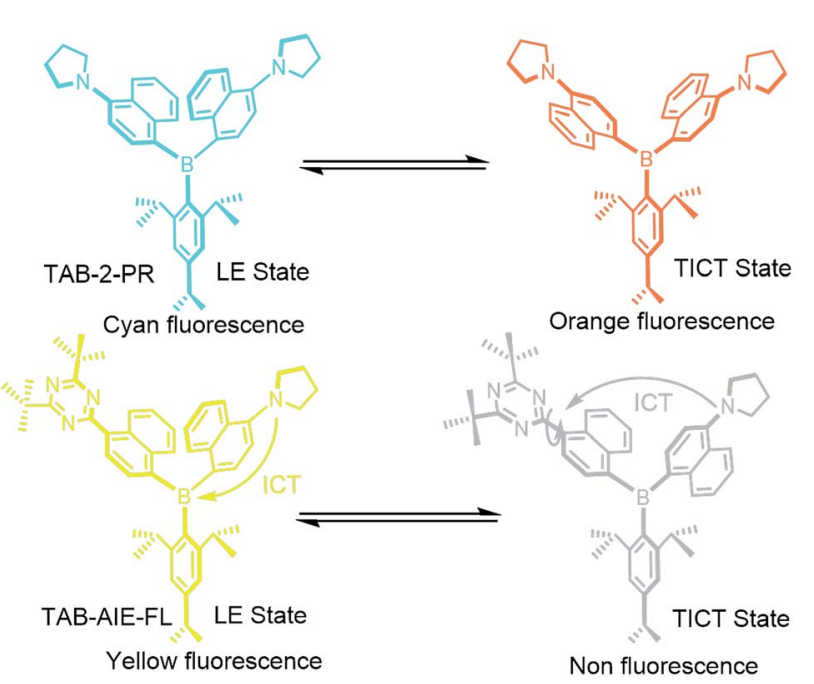

Scheme 1 The mechanism of emission changes of TAB-2-PR and TAB-AIE-FL in LE state and TICT state, respectively.
(Fig. S1 $\uparrow$ and $1 \mathrm{a}$ and $\mathrm{b}$ ). Their maximum absorptions appear at wavelengths of around $400 \mathrm{~nm}$, which indicate that they have the same degree of conjugation. TAB-2-PR demonstrates strong fluorescence in various solvents and its fluorescence spectra show significant bathochromic shifts with increasing solvent polarity, which indicates that TAB-2-PR bears typical ICT characteristics as these triarylboron compounds that we investigated before. However, TAB-AIE-FL on replacing one of two pyrrolidine groups in TAB-2-PR with 2,4-di-tert-butyl-s-triazine shows strong fluorescence in the solid state and in apolar hexane, but almost no fluorescence in polar solvents. The emission peak of TAB-AIE-FL in $n$-hexane appears at $575 \mathrm{~nm}$, a large bathochromic shift compared with TAB-2-PR at $450 \mathrm{~nm}$, which implies that the 2,4-di-tert-butyl-s-triazine group indeed increases the ICT and results in a larger Stokes shift (Fig. S2 $\dagger$ ). The fluorescence quantum yields of TAB-AIE-FL were also further determined to be 0.403 in the solid state, 0.121 in apolar $n$-hexane and almost zero in other polar solvents on a Hamamatsu absolute PL quantum yield spectrometer C11347 (Table $\mathrm{S} 1 \dagger)$. The high fluorescence quantum yield of TAB-AIE-FL in the solid state compared with apolar $n$-hexane results from the restricted rotation of 2,4-di-tert-butyl-s-triazine. The strong emission of TAB-AIE-FL in the apolar solvent can be attributed to the LE state, which can be stabilized by the apolar environment. ${ }^{20}$ However, the intramolecular twisting in the polar solvent transforms TAB-AIE-FL from the LE state to the TICT state, where a total charge separation between its D and A units occurs. The fluorescence is completely quenched due to the rotation of 2,4-di-tert-butyl-s-triazine and the susceptibility of the TICT state to non-radiative decay processes.

Since TAB-AIE-FL is water-insoluble, the addition of a certain amount of water into the solution of TAB-AIE-FL in DMSO can induce the formation of aggregates. Therefore, the AIE properties of TAB-AIE-FL can also be confirmed by measuring its fluorescence spectra change during this process. In Fig. 2a, the emergence of a typical tail absorbance in the long-wavelength region implies the formation of aggregates. Fig. $2 b$ further shows TAB-AIE-FL experiences a large enhancement of fluorescence after aggregating, clearly manifesting its AIE activity. It is generally thought that there are two main reasons that can contribute to the AIE process of TICT luminogens. First, instead of the emission behavior in polar solvents, the formation of aggregates can inhibit the dark TICT process and ensure the emission of the highly efficient LE state. TAB-AIE-FL, bearing the two tert-butyls with large steric hindrance in its molecular
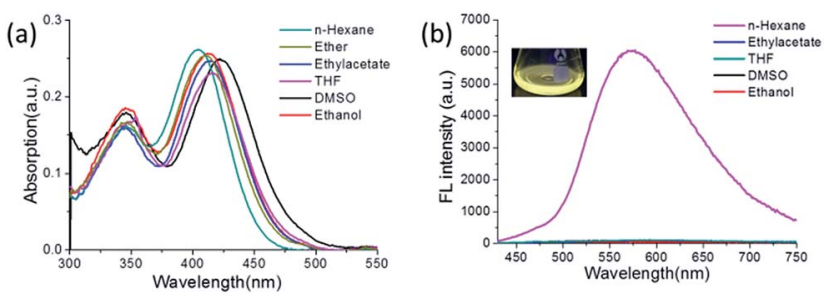

Fig. 1 (a) UV absorption and (b) fluorescence spectra of TAB-AIE-FL in different solvents $(10 \mu \mathrm{M})$. Excitation wavelength: $415 \mathrm{~nm}$. 

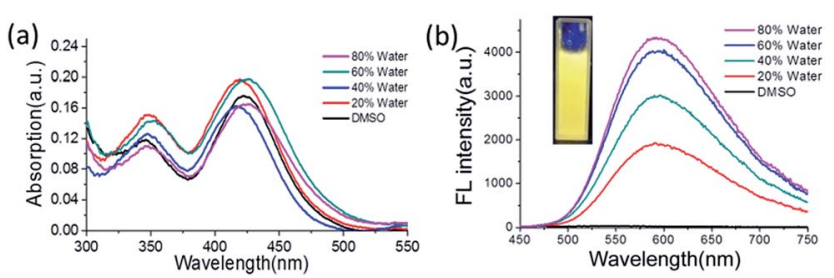

Fig. 2 (a) UV absorption and (b) fluorescence spectra of TAB-AIE-FL with the formation of aggregates in different ratios of the mixed solvents of water and DMSO $(10 \mu \mathrm{M})$. Excitation wavelength: $415 \mathrm{~nm}$.

structure, can block $\pi-\pi$ stacking interaction in the aggregates and prevent the short-range interchromophoric energy migration and transfer. ${ }^{9}$ Second, once aggregation occurs, the vigorous rotation of the triazine part can be restricted and the corresponding non-radiative pathway can be hindered. Therefore, the cooperation of the inhibition of TICT and restriction of intramolecular motion results in the large enhancement of fluorescence, as shown in Fig. 2b. The UV absorption and fluorescence spectra of TAB-AIE-FL in the solid state are also recorded in Fig. S3. $\dagger$

In order to better understand the AIE mechanism of TABAIE-FL, a density functional theory (DFT) computation experiment was conducted using the Gaussian09 program. From the calculations, two stable molecular geometries are found both for TAB-2-PR (1 and 1X) and TAB-AIE-FL (2 and 2X) in the ground state by changing the dihedral angle labeled in cyan in Fig. S4. $\dagger$ Correspondingly, in the lowest singlet excited state, there also exist two stable geometries for TAB-2-PR ( $1^{*}$ and $\left.1 \mathrm{X}^{*}\right)$ and TAB-AIE-FL ( $2 *$ and $\left.2 X^{*}\right)$, as shown in Fig. 3 and S5 and S6. $\dagger$ From the electronic cloud distribution of the frontier orbitals of TAB-2-PR, it is clearly seen that the naphthalene rings and pyrrole groups play the role of electron donors and the boron atom acts as an electron acceptor. In contrast, for the two ground states and excited states of TAB-AIE-FL, the electronic cloud distribution of LUMO orbitals are mainly centered around the triazine group, which implies that the triazine group is a more strong electron acceptor than the boron atom and can boost a complete charge separation. The configuration of 2 tends to be more planar than $2 \mathrm{X}$. By comparing the HOMO orbitals of 2 and $2 \mathrm{X}$, it can be found that there is a certain distribution of electronic cloud around the naphthalene rings conjugated with triazine in 2 while hardly any distribution of

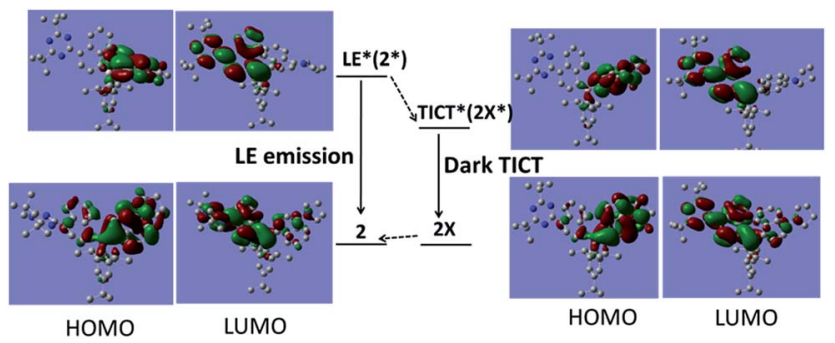

Fig. 3 The frontier orbital electron cloud distributions of the LE state and the TICT state of TAB-AIE-FL. electronic cloud in $2 \mathrm{X}$, which also proves the total complete charge separation in $2 \mathrm{X}$. Thus, the emissive $\mathrm{LE}^{*}$ and the nonemissive TICT* originate from $2^{*}$ and $2 \mathrm{X}^{*}$, respectively. Our calculations show the energy of configuration $2 \mathrm{X}^{*}$ is $1.17 \mathrm{eV}$ lower than that of $2^{*}$, which indicates that the transition from the $\mathrm{LE}^{*}\left(2^{*}\right)$ to the $\mathrm{TICT}^{*}\left(2 \mathrm{X}^{*}\right)$ is possible. In polar solvents, since TAB-AIE-FL molecules are unrestricted and can freely rotate, the molecularly dispersed solutions are almost not emissive. In apolar solvents, the planar configuration of 2 can be partially stabilized and, therefore, a certain intensity of fluorescence is observed. In the solid state, due to the complete restriction of rotation and the transition from the $\mathrm{LE}^{*}\left(2^{*}\right)$ to the TICT* $\left(2 X^{*}\right)$, TAB-AIE-FL exhibits strong fluorescence.

Considering that high viscosity can restrict the intramolecular motion and further restrict the formation of TICT, the investigation of the effect of viscosity on the fluorescence spectra of TAB-AIE-FL can help us better understand its AIE mechanism and also explore its application as a fluorescence probe of viscosity. Therefore, we measured the fluorescence spectra of TAB-AIE-FL in mixed solvents of glycerol and methanol with different ratios. Since the polarity of glycerol and methanol differs greatly, it is difficult to exclude the influence of polarity during the experiment. Fig. 4 shows the comprehensive effect of viscosity and polarity on the UV absorption and fluorescence spectra of monodispersed TAB-AIE-FL. When the proportion of glycerol is less than $80 \%$, the fluorescence intensity of TAB-AIE-FL increases with the increase in the ratio of glycerol, which can be attributed to the dominant effect of the increasing viscosity (Fig. $4 \mathrm{~b}$ and c). However, once the ratio of glycerol exceeds $80 \%$, the increase of polarity plays a leading role and the fluorescence intensity of TAB-AIE-FL decreases with the increase of glycerol ratio. The fluorescence lifetime of TABAIE-FL is shorter in aggregate state than in monodispersed state (Fig. 4d). The abovementioned results may provide more evidence about the reasonable explanation for the AIE mechanism of TAB-AIE-FL by the restriction of TICT process and intramolecular motions in the aggregate state and also show that TAB-AIE-FL may be potential useful as a "turn on" fluorescent sensor for viscosity.
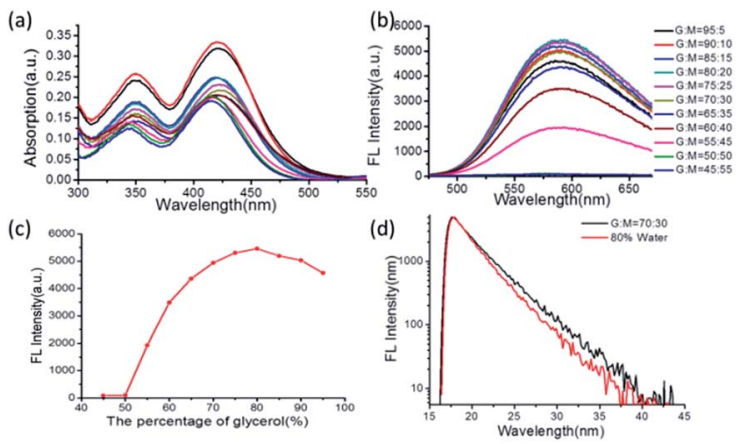

Fig. 4 (a) UV absorption and (b) fluorescence spectra of TAB-AIE-FL with the formation of aggregates in different ratios of the mixed solvents of glycerol and methanol (G: M) $(10 \mu \mathrm{M})$. (c) A correlation between the percentage of glycerol and fluorescence intensity. (d) Fluorescence decay of TAB-AIE-FL in aggregate state and monodispersed state. Excitation wavelength: $415 \mathrm{~nm}$. 
(a)

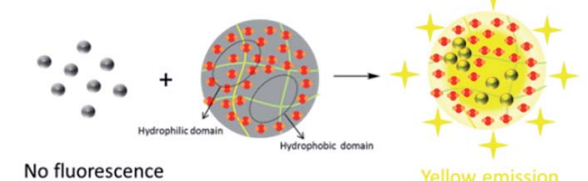

(b)

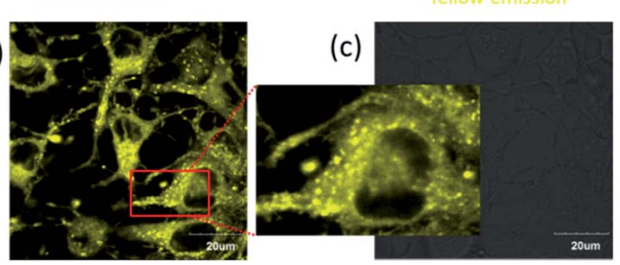

Fig. 5 (a) The mechanism of strong emission of TAB-AIE-FL incorporated in the nanogel. Confocal fluorescence images of NIH/3T3 cells incubated with NG-TAB-FL for $30 \mathrm{~min}$ (b) and corresponding bright field (c).

In order to demonstrate the application of TAB-AIE-FL in biological imaging, we chose our previously studied polyurethane hydrogel as the carrier for TAB-AIE-FL and prepared the nanogel-bearing TAB-AIE-FL (NG-TAB-AIE-FL) to improve its water-solubility and biocompatibility ${ }^{26}$ (Fig. 5a). The average diameter of NG-TAB-AIE-FL particles was measured as $295 \mathrm{~nm}$ by dynamic light scattering (DLS) (Fig. S11†). Since the nanogel contains both hydrophilic and hydrophobic domains, waterinsoluble TAB-AIE-FL will mainly distribute in the apolar hydrophobic domains with single molecules or in the hydrophilic domains with aggregates, both of which can show strong fluorescence. The high viscosity of the nanogel interior also can enhance the fluorescence of TAB-AIE-FL. Therefore, NG-TABAIE-FL demonstrates strong fluorescence. Moreover, we introduced NG-TAB-AIE-FL into NIH/3T3 cells (Beijing Union Medical College Hospital, China) and realized the imaging by confocal fluorescence microscopy. The results show that the NG-TAB-AIE-FL demonstrates strong fluorescence in the cytoplasm and some small aggregates (1-3 $\mu \mathrm{m}$ in diameter) can be clearly seen, which show that TAB-AIE-FL may be eluted from the nanogel interior after entering the cell and aggregate again in the hydrophilic environment of cytoplasm (Fig. 5b and c). Interestingly, in the nucleus, there are no aggregates, but bright fluorescence is present. Combining with our previous reports that the nanogel can only distribute in the cytoplasm, ${ }^{\mathbf{1 1 2 6}}$ we deduced that the fluorescence may result from the eluted TABAIE-FL, which is in the highly viscous and low polar environment of the nucleus.

\section{Conclusion}

By introducing a triazine group into the molecular structure of triarylboron, a triarylboron-based AIE fluorophore, TAB-AIE-FL, was designed and synthesized. Its AIE properties were further confirmed by some experimental data. Theoretical investigations also indicate that there exist two excited states for TABAIE-FL corresponding to its LE excited state and nonemissive TICT excited state. We also explore its application as a fluorescence sensor for viscosity. By utilizing nanogel as a carrier, TAB-
AIE-FL was successfully introduced into NIH/3T3 cells for imaging the hydrophilic environment of the cytoplasm and the highly viscous and low polar intracellular environment of nucleus. Such a success for endowing the triarylboron compound, an ACQ fluorophore, with AIE activity can expand its application as a novel fluorophore in fields such as biology, optics and electronics.

\section{Acknowledgements}

We are grateful for the funding from the National Natural Science Foundation of China (Grant No. 21172025, 8167175, 21503026), the Bureau of Science \& Technology and Intellectual Property Nanchong City (Grant No. 16YFZJ0121) and the North Sichuan Medical College (Grant No. CBY16-QD-01).

\section{Notes and references}

1 F. Jäkle, Chem. Rev., 2010, 110, 3985-4022.

2 C. R. Wade, A. E. J. Broomsgrove, S. Aldridge and F. P. Gabbai, Chem. Rev., 2010, 110, 3958-3984.

3 A. Ito, K. Kawanishi, E. Sakuda and N. Kitamura, Chem.-Eur. J., 2014, 20, 3940-3953.

4 C. Hoffend, F. Schodel, H. W. Lerner and M. Wagner, Chem.Eur. J., 2012, 18, 15394-15405.

5 V. M. Hertz, M. Bolte, H. W. Lerner and M. Wagner, Angew. Chem., Int. Ed., 2015, 54, 8800-8804.

6 J. Feng, K. Tian, D. Hu, S. Wang, S. Li, Y. Zeng, Y. Li and G. Yang, Angew. Chem., Int. Ed., 2011, 50, 8072-8076.

7 X. Liu, S. Li, J. Feng, Y. Li and G. Yang, Chem. Commun., 2014, 50, 2778-2780.

8 S. M. Griesbeck, Z. Zhang, M. Gutmann, T. Lühmann, R. M. Edkins, G. Clermont, A. N. Lazar, M. Haehnel, K. Edkins and A. Eichhorn, Chem.-Eur. J., 2016, 22, 1470114706.

9 X. Li, X. Guo, L. Cao, Z. Xun, S. Wang, S. Li, Y. Li and G. Yang, Angew. Chem., Int. Ed., 2014, 53, 7809-7813.

10 X. Guo, X. Zhang, S. Wang, S. Li, R. Hu, Y. Li and G. Yang, Anal. Chim. Acta, 2015, 869, 81-88.

11 J. Liu, X. Guo, R. Hu, J. Xu, S. Wang, S. Li, Y. Li and G. Yang, Anal. Chem., 2015, 87, 3694-3698.

12 J. Liu, X. Guo, R. Hu, X. Liu, S. Wang, S. Li, Y. Li and G. Yang, Anal. Chem., 2016, 88, 1052-1057.

13 C. Zhang, Y. Yuan, S. Zhang, Y. Wang and Z. Liu, Angew. Chem., Int. Ed., 2011, 50, 6851-6854.

14 J. Luo, Z. Xie, J. W. Lam, L. Cheng, X. Zhan, Y. Liu, D. Zhu and B. Z. Tang, Chem. Commun., 2001, 18, 1740-1741.

15 Y. N. Hong, J. W. Y. Lam and B. Z. Tang, Chem. Commun., 2009, 18, 4332-4353.

16 J. Mei, N. L. Leung, R. T. Kwok, J. W. Lam and B. Z. Tang, Chem. Rev., 2015, 115, 11718-11940.

17 C. W. Leung, Y. Hong, S. Chen, E. J. Zhao, W. Lam and B. Z. Tang, J. Am. Chem. Soc., 2013, 135, 62-65.

18 Z. Wang, S. Chen, J. W. Lam, W. Qin, R. T. Kwok, N. Xie, Q. Hu and B. Z. Tang, J. Am. Chem. Soc., 2013, 135, 82388245 . 
19 D. Ding, K. Li, B. Liu and B. Z. Tang, Acc. Chem. Res., 2013, 46, 2441-2453.

20 J. Mei, Y. Hong, J. W. Lam, A. Qin, Y. Tang and B. Z. Tang, Adv. Mater., 2014, 26, 5429-5479.

21 J. W. Li, Y. Qian, L. H. Xie, Y. P. Yi, W. W. Li and W. Huang, J. Phys. Chem. C, 2015, 119, 2133-2141.

22 R. Hu, E. Lager, A. Aguilaraguilar, J. Liu, K. S. Wong and E. Peñacabrera, J. Phys. Chem. C, 2009, 113, 15845-15853.
23 B. R. Gao, H. Y. Wang, Z. Y. Yang, H. Wang, L. Wang, Y. G. Ma and H. B. Sun, J. Phys. Chem. C, 2011, 115, 1615016154.

24 W. Qin, D. Ding, J. Liu, W. Z. Yuan, Y. Hu, B. Liu and B. Z. Tang, Adv. Funct. Mater., 2012, 22, 771-779.

25 S. Kim, Q. Zheng, G. S. He, D. J. Bharali, H. E. Pudavar, A. Baev and P. N. Prasad, Adv. Funct. Mater., 2006, 16, 2317-2323.

26 L. Cao, X. Li, S. Wang, S. Li, Y. Li and G. Yang, Chem. Commun., 2014, 50, 8787-8790. 